\title{
APLICAÇÃO DE POLÍMEROS NA FLOCULAÇÃO DE REJEITO DE CAULIM: EFEITOS DA VARIAÇÃO DE CONCENTRAÇÃO E TIPO DE FLOCULANTE
}

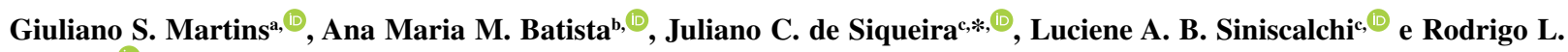 \\ Oréfice $^{\mathrm{d}, \text { (D) }}$ \\ aDepartamento de Física, Instituto Federal de Minas Gerais, Campus Congonhas, 36415-000 Congonhas - MG, Brasil \\ bDepartamento de Recursos Naturais, Ciências e Tecnologia Ambiental, Universidade do Estado de Minas Gerais, Campus João \\ Monleavade, 35930-314 João Monlevade - MG, Brasil \\ 'Departamento de Engenharia Ambiental, Universidade Federal de Lavras, 37200-900 Lavras - MG, Brasil \\ ${ }^{\mathrm{d}}$ Departamento de Engenharia Metalúrgica e de Materiais, Universidade Federal de Minas Gerais, 31270-900 Belo Horizonte - \\ MG, Brasil
}

Recebido em 25/10/2021; aceito em 07/12/2021; publicado na web em 04/02/2022

\begin{abstract}
APPLICATION OF POLYMERS IN THE FLOCCULATION OF KAOLIN WASTE: EFFECTS OF VARYING CONCENTRATION AND TYPE OF FLOCCULANT. This study assessed the effects of volume concentration $(0.3 \%, 0.6 \%, 1.2 \%$, and $12 \%$ v/v) and type of polymer flocculant (acrylamide, chitosan, and purified and non-purified acrylamide grafted chitosan) on the flocculation mechanism of kaolinite-rich mining tailings samples. We measured the flocculated samples' transmittance using UV-VIS spectrometry to check for particles in the supernatant medium in order to verify the flocculation efficiency. It was observed that flocculation directly depends on the $\mathrm{pH}$ of the medium, the isoelectric point of the kaolinite, and the polymer chains' molecular mass. The concentration of $\mathrm{H}^{+}$and $\mathrm{OH}^{-}$ions is responsible for producing charges of different electrical nature on the particles present in the suspension, thereby affecting the flocculation process. In the acrylamide samples, the aggregation of particles occurs by electrostatic interaction; and in the other samples by bridging, suggesting that the chains' molecular mass interferes in the coagulation and flocculation mechanisms. Moreover, the results of absorbance measurements and flocs' apparent density highlight that the grafting of acrylamide on the chitosan chain may improve the kaolin waste flocculation. For flocculant concentrations of up to $1.2 \%(\mathrm{v} / \mathrm{v})$, the absorbances were mostly below 1.0 , indicating removal of suspended materials.
\end{abstract}

Keywords: chitosan; natural flocculant; acrylamide; biopolymer; chemical functionalization.

\section{INTRODUÇÃO}

Embora a Terra seja formada por $75 \%$ de água, os recursos hídricos disponíveis para consumo humano, tais como saneamento, agricultura e indústria, são escassos. ${ }^{1,2}$ A redução dos recursos hídricos e o aumento da população mundial revelam a importância da utilização de técnicas para manutenção, reaproveitamento e tratamento da água. ${ }^{3}$

Nesse sentido, os processos de tratamento envolvem a remoção de contaminantes e partículas que variam de acordo com o meio a ser tratado. Os efluentes industriais, por exemplo, contêm partículas sólidas com uma infinidade de formas, tamanhos, densidades e composição que alteram o comportamento da fase líquida e a capacidade de remoção de poluentes. Os contaminantes químicos e microbiológicos podem ser adsorvidos à essas partículas, fazendo-se necessário a remoção destes materiais. ${ }^{4} \mathrm{~A}$ retirada de contaminantes pode ser realizada, por exemplo, por gravidade (sedimentação), coagulação e floculação. ${ }^{1,5,6}$

A separação de partículas sólidas por gravidade é realizada através de sedimentação. Essas partículas possuem normalmente densidade maior que da água e, assim, sedimentam. Entretanto, a eficiência do processo é limitada ao tamanho das partículas. O tempo de sedimentação aumenta com a redução do tamanho, tornando o processo economicamente inviável em algumas aplicações. ${ }^{7}$

Por outro lado, o processo de coagulação consiste na desestabilização de suspensões coloidais através da neutralização das forças elétricas que mantêm as partículas suspensas separadas. Os agregados formados no processo de coagulação são pequenos e fracamente

*e-mail: julianocuri2015@gmail.com ligados, dessa forma a velocidade de sedimentação é relativamente baixa. O processo é altamente dependente de variações de $\mathrm{pH}^{8}$

A floculação, por sua vez, é realizada pela adição de químicos de origem orgânica ou inorgânica no líquido. O mecanismo de floculação altera-se de acordo com o material utilizado no processo. Os floculantes inorgânicos são normalmente formados por sais de metais multivalentes como alumínio e ferro. O emprego de floculantes inorgânicos demanda alta quantidade de material e é afetado por mudanças no $\mathrm{pH}^{8,9}$

Os floculantes orgânicos, por outro lado, são formados por polímeros naturais e sintéticos. Os polímeros naturais são biodegradáveis e estáveis sob cisalhamento, porém, exigem altas concentrações para efetiva atividade de floculação. Além do mais, são seguros se comparados com floculantes químicos convencionais, disponíveis para aplicações na agricultura e não produzem poluição secundária. ${ }^{10,11}$

Os polímeros sintéticos, por sua vez, necessitam de baixas concentrações (da ordem de ppm), podendo ser feitos sob medida, controlando-se seu peso molecular e estrutura química, bem como a natureza e a proporção dos grupos funcionais na cadeia do material. Todavia, são poucos resistentes ao cisalhamento e não sofrem degradação biológica. ${ }^{1,12}$

Desse modo, o interesse no uso de floculantes biodegradáveis de origem natural tem se intensificado. Polissacarídeos naturais podem ser empregados, tais como amido, goma de guar, alginato, dextrano, glicogênio e quitina. ${ }^{13} \mathrm{~A}$ modificação química desses materiais visando melhorar a eficácia da floculação é possível através da enxertia nas cadeias de polissacarídeos de porções catiônicas. ${ }^{14} \mathrm{Os}$ materiais enxertados emergiram com grande potencial para tratamento de águas residuárias devido às propriedades específicas e superiores em relação aos materiais convencionais. ${ }^{15}$ 
Nesse sentido, floculantes baseados em polissacarídeos exibem maior estabilidade dos flocos e maior degradabilidade em meio aquoso. A modificação de polímeros naturais tem sido explorada no sentido de melhoria das propriedades com a incorporação de polímeros sintéticos para aumentar o poder de floculação. ${ }^{16} \mathrm{~A}$ enxertia de monômeros vinílicos em polímeros de quitosana, por exemplo, pode introduzir novas propriedades e aumentar suas aplicações através da adição de grupos laterais na cadeia do polímero. ${ }^{17}$

A literatura descreve os processos de coagulação e floculação através de diversos mecanismos que envolvem interações eletrostáticas, desestabilização de superfícies de cargas, ponteamento entre amostras poliméricas e materiais agregados. ${ }^{18,19}$

Neste trabalho, estudou-se o efeito da variação de concentração em volume $(0,3 \%, 0,6 \%, 1,2 \%$ e $12 \%$ v/v) e tipo de floculante (quitosana, acrilamida, quitosana enxertada com acrilamida purificada e quitosana enxertada com acrilamida não purificada) no mecanismo de floculação de amostras ricas em caulinita.

\section{PARTE EXPERIMENTAL}

\section{Materiais}

Para enxertia do polímero foram empregados os seguintes reagentes: quitosana e acrilamida adquiridos pela Sigma-Aldrich ${ }^{\circledR}$; e ácido acético, hidróxido de sódio, etanol, persulfato de sódio adquiridos pela $S y n t h^{\circledast}$. Todos os reagentes foram empregados sem preparo prévio.

As amostras de rejeito foram obtidas a partir do rejeito de mineração da extração de minerais não metálicos provenientes da região de Esmeraldas, Minas Gerais, Brasil.

\section{Produção dos floculantes}

\section{Enxertia de acrilamida no polímero de quitosana}

O processo de enxertia foi realizado tomando-se como referência o método proposto por Zhang et al: $:^{20}$ na primeira etapa do processo, 0,5 g de quitosana (grau de deacetilação 75\%, massa molar $\approx 33,61 \times 10^{4} \mathrm{~g} \mathrm{~mol}^{-1}$ ) foi dissolvida em uma solução aquosa de ácido acético (1 wt\%). Posteriormente, adicionou-se $5 \mathrm{~mL}$ de solução aquosa de persulfato de sódio $(0,28 \mathrm{wt} \%)$ para formação de radicais livres. A mistura foi mantida sob temperatura de $60^{\circ} \mathrm{C}$ e agitação de 100 rpm durante $1 \mathrm{~h}$ (etapa i, Figura 1) em um balão de três pescoços equipado com um misturador e um termômetro.

Realizou-se a enxertia dos radicais acrilatos na estrutura da quitosana (etapa ii, Figura 1). O processo iniciou-se com adição de $8 \mathrm{~mL}$ de solução aquosa de acrilamida (33 wt\%) na solução contendo quitosana. Os reagentes foram mantidos sob agitação de 40 rpm durante $3 \mathrm{~h}$ e temperatura de $60^{\circ} \mathrm{C}$. Em seguida, o material foi resfriado até a temperatura ambiente $\left(\approx 25^{\circ} \mathrm{C}\right)$. A acidez do meio foi neutralizada empregando-se uma solução aquosa de $\mathrm{NaOH}$ (20 wt \%) até pH 7,0. A solução foi então lavada e precipitada com etanol e posteriormente filtrada com funil de Büchner. Posteriormente, para obtenção de um polímero com alta pureza, o material foi filtrado novamente utilizando-se um Soxhlet contendo etanol. Finalmente, o resultado do processo de filtração foi secado em uma estufa a vácuo durante $48 \mathrm{~h}$ e temperatura de $60{ }^{\circ} \mathrm{C}$ para retirada da umidade residual e possíveis sólidos voláteis oriundos do processo de enxertia. As amostras obtidas a partir desse processo foram denominadas Quitosana+Acrilamida (purificada) (etapa iii, Figura 1).

Para estudar os efeitos das variáveis de processo, um grupo de amostras foi produzido sem passar pelas etapas de neutralização da

i)

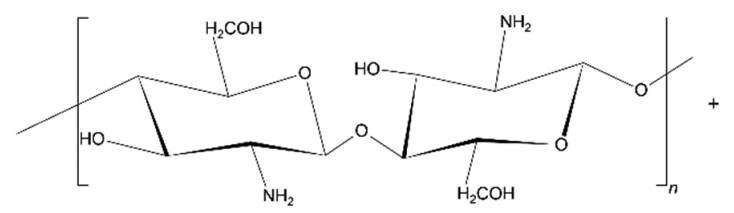

Quitosana

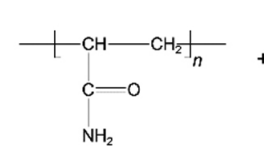

Acrilamida

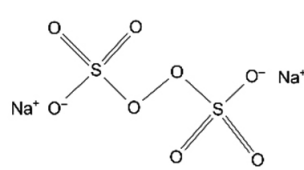

Persulfato de sódio

ii)

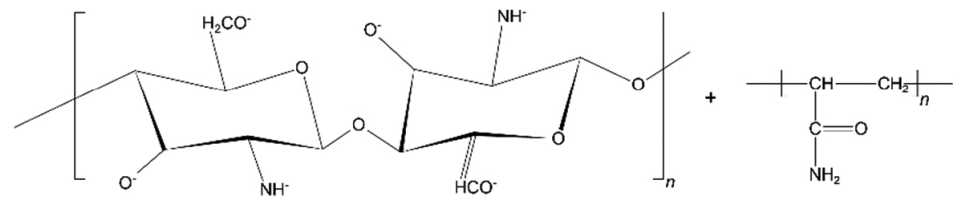<smiles>CC(C)C(N)=O</smiles>

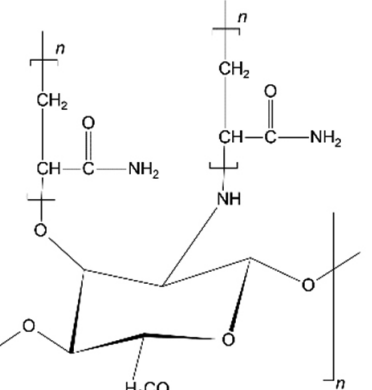


acidez com adição de $\mathrm{NaOH}$ e filtração via funil de Büchner e Soxhelt, sendo denominadas Quitosana+Acrilamida.

\section{Floculante de quitosana}

O floculante de quitosana foi obtido a partir da dissolução de $1 \mathrm{~g}$ de quitosana em $100 \mathrm{~mL}$ de solução aquosa de ácido acético (1 wt \%).

\section{Floculante de acrilamida}

O floculante foi obtido a partir da dissolução da acrilamida em água, empregando-se concentração de 33 wt\%.

\section{Procedimento experimental}

\section{Difração de raios $X$ - Caracterização do rejeito}

A identificação das fases cristalinas do rejeito foi realizada por meio de difratometria de raios $\mathrm{X}$ pelo método do pó. Utilizou-se um difratômetro de fabricação Rigaku ${ }^{\circledR}$, modelo D $D A X$ ÚLTIMA automático, com goniômetro $\theta-\theta$ e tubo de raios $X$ de cobre.

As leituras foram realizadas na faixa de $2 \theta\left(4\right.$ a $\left.80^{\circ}\right)$, velocidade do goniômetro $\left(1^{\circ} \mathrm{min}^{-1}\right)$, intensidade de corrente $(30 \mathrm{~mA})$ e tensão $(40 \mathrm{kV})$.

A identificação dos minerais foi realizada por comparação do difratograma de raios $\mathrm{X}$ das amostras com o banco de dados da International Center for Diffraction Data (ICDD) / Joint Committee on Powder Diffraction Standards (JCPDS) (Sets 01 - 58; MDI; Jade 9; 2008).

A difração de raios $X$ foi importante para a detecção da composição mineral do meio empregado no presente estudo, com a hipótese de uma maior concentração de caulinita no rejeito.

\section{Espectroscopia de infravermelho}

A espectroscopia de infravermelho (FTIR) foi empregada para verificação da formação de ligações químicas provenientes do processo de enxertia. $\mathrm{O}$ espectro de infravermelho foi coletado utilizando espectroscopia de infravermelho com transformada de Fourier (FTIR, Nicolet model 6700) e Reflexão Total Atenuada (ATR). Cada espectro foi obtido empregando-se 64 varreduras, resolução espectral de $4 \mathrm{~cm}^{-1}$ na faixa de 650 a $4000 \mathrm{~cm}^{-1}$.

A aplicação da FTIR foi relevante para o presente estudo pois permitiu evidenciar a formação de estruturas poliméricas durante o processo de enxertia dos materiais, garantindo que a técnica foi empregada corretamente.

\section{Teste de floculação}

O teste de floculação foi realizado empregando-se amostras de rejeito de mineração ricas em caulim. O rejeito foi misturado com as amostras de floculante (quitosana, acrilamida, quitosana enxertada com acrilamida purificada e quitosana enxertada com acrilamida não purificada) obedecendo-se as concentrações de 0,3, 0,6, 1,2 e $12 \%$ v/v. O salto no valor de concentração volumétrica apresentado $(1,2 \%$ para $12 \% \mathrm{v} / \mathrm{v})$ visou evidenciar o comportamento das amostras a partir do respectivo aumento, no sentido de indicar a existência de um valor de concentração limítrofe para cada floculante aplicado no presente estudo.

Em seguida, o material foi agitado por 5 min utilizando-se rotação de $40 \mathrm{rpm}$ para promover o crescimento dos flocos.

\section{Análise da turbidez}

Para verificar a dinâmica de floculação, foram coletadas imagens do processo de formação de flocos após 4 min contados a partir do término da agitação mecânica. As imagens foram utilizadas para análise qualitativa do processo de formação de flocos e para estimar a densidade aparente dos flocos formados.
O sobrenadante foi coletado depois de 5 min de repouso e a porcentagem de transmitância foi medida (\%T) a $550 \mathrm{~nm}$ usando um espectrofotômetro (Shimadzu, UV 2600).

\section{Densidade aparente dos flocos depositados}

A densidade aparente dos flocos foi determinada medindo-se a altura das colunas de material depositados (material floculado) no fundo dos béqueres, juntamente com as dimensões do recipiente, determinando-se assim o volume ocupado pelos flocos. A massa de material depositado foi obtida retirando-se o material sobrenadante e evaporando-se o restante do líquido. Com a razão massa/volume, determinou-se o valor de densidade aparente para os flocos.

\section{RESULTADOS E DISCUSSÃO}

\section{Espectroscopia de infravermelho}

O resultado das medidas de FTIR realizadas com quitosana e quitosana enxertada com acrilamida é apresentado na Figura 2.

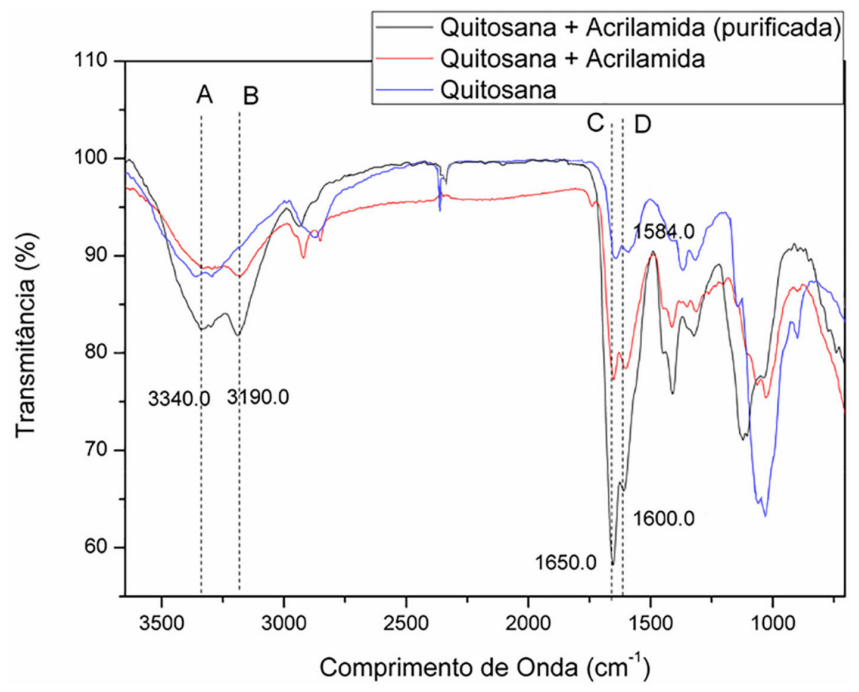

Figura 2. FTIR realizada com amostras de quitosana pura (curva de cor azul) e quitosana enxertada com acrilamida não purificada (curva de cor vermelha) e purificada (curva de cor preta)

O processo de enxertia foi avaliado por meio de espectroscopia de infravermelho. As bandas em $1650 \mathrm{~cm}^{-1}$ e $1584 \mathrm{~cm}^{-1}$ (retas C e $\mathrm{D}$, respectivamente) detectadas nas amostras de quitosana pura são associadas às vibrações do grupamento $\mathrm{NH}_{2}$. Nas amostras nas quais a acrilamida foi enxertada, observam-se, em $1600 \mathrm{~cm}^{-1}$, bandas associadas às vibrações de dobramento do grupo $\mathrm{NH}$. Os grupos NH são formados a partir da ligação covalente entre um carbono insaturado presente na acrilamida e o grupamento $\mathrm{NH}_{2}$ presente na quitosana. Somando-se a isso, o aumento da intensidade da banda a $1650 \mathrm{~cm}^{-1}$ sugere aumento da absorção provocada por grupos $\mathrm{NH}_{2}$ provenientes de ligações do tipo $-\mathrm{CO}-\mathrm{NH}_{2}$. As alterações na intensidade de absorção a $1650 \mathrm{~cm}^{-1} \mathrm{e}$ o surgimento da banda em 1600 $\mathrm{cm}^{-1}$ sugerem que acrilamida foi enxertada na cadeia da quitosana.

A variação da intensidade e o deslocamento na banda próxima a $3400 \mathrm{~cm}^{-1}$ (vibração de alongamento dos grupos $\mathrm{OH}$ e $\mathrm{NH}$ ) indicam que grupamentos $\mathrm{OH}$ e $\mathrm{NH}$ da quitosana participaram da reação de enxertia. O desvio para $3340 \mathrm{~cm}^{-1}$ (blue-shift, reta A) e o aumento da intensidade relativa da absorção nesse comprimento de onda indica a formação de grupos amidas entre a quitosana e acrilamida. A banda encontrada a $3190 \mathrm{~cm}^{-1}$ (reta B) é associada aos grupos aminas livres provenientes da acrilamida. 


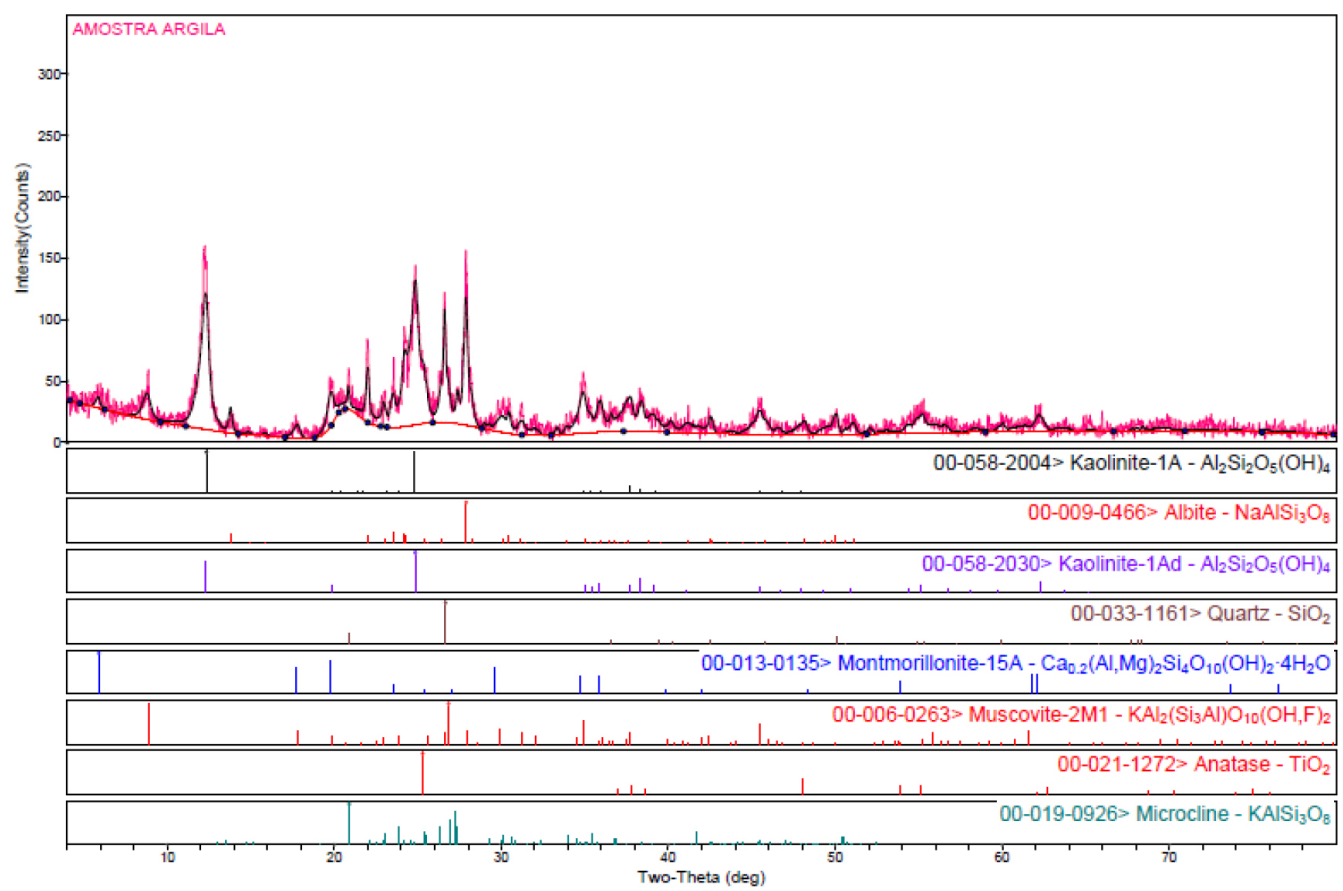

Figura 3. Difratograma obtido a partir das amostras de rejeito, destacando a composição mineral

\section{Caracterização das amostras de rejeito}

\section{Difração de raios $X$}

A Figura 3 apresenta o difratograma obtido a partir das amostras de rejeito.

Observa-se a partir dos ensaios de difração de raios $\mathrm{X}$ que as amostras analisadas contêm caulinita. A caulinita é um aluminossilicato com dois planos basais de clivagem: uma folha octaédrica de alumina e uma folha tetraédrica de sílica. ${ }^{21}$ Essas folhas são ligadas por átomos de oxigênio compartilhados. Entre as camadas, as ligações são formadas por interações eletrostáticas, forças de Van der Waals e ligações de hidrogênio. ${ }^{22}$ As bordas das camadas expostas de $\mathrm{Al}$ e Si são terminadas por grupos hidroxilas. ${ }^{23}$

Segundo Hsua et al. ${ }^{24}$ diferentes cargas de superfície contribuem para a carga superficial total de materiais argilosos. A contribuição para a carga total é originária de substituições isomórficas ou quebra de ligações, íons $\mathrm{H}^{+}$ou $\mathrm{OH}^{-}$e cargas resultantes de íons adsorvidos na camada de Stern.

De acordo com Brady et al.,${ }^{25}$ a carga superficial da caulinita sofre alterações de acordo com a composição química do meio e defeitos estruturais. As faces das camadas de alumina estão sujeitas a protonação e deprotonação através da formação de íons $\mathrm{OH}^{2}, \mathrm{OH}^{-} \mathrm{e}$ $\mathrm{O}^{-2}$, de acordo com o $\mathrm{pH}$ do meio. As extremidades da camada estão sujeitas a formação dos mesmos íons por protonação, deprotonação e quebra de ligações. As extremidades das camadas de Si apresentam comportamento semelhante às extremidades das camadas de $\mathrm{Al}$, apresentando alta dependência com o pH do meio. Por outro lado, as faces das camadas de sílica estão sujeitas às substituições isomórficas, adquirindo sempre carga negativa e pouca dependência com o $\mathrm{pH}$ do meio. ${ }^{26-28}$

A superfície da carga da caulinita é altamente dependente da relação entre o ponto isoelétrico da partícula e o pH do meio. Carty propôs que a caulinita tem superfície de carga da alumina positiva, enquanto que a sílica tem carga negativa na faixa de $\mathrm{pH}$ de 3,5 a 8,5, baseado no ponto isoelétrico da sílica $(2,5$ a 3,5$)$ e da alumina $(8,5$ a 10,4$).{ }^{21}$ De modo geral, a carga total dos planos basais é sempre negativa, mas a magnitude é dependente do $\mathrm{pH} .{ }^{29,30} \mathrm{Em}$ valores de $\mathrm{pH}$ abaixo do ponto isoelétrico, o material sofre protonação; e acima do ponto isoelétrico, deprotonação (Figura 4). Quando o valor é igual ao ponto isoelétrico, existe a formação dos dois tipos de carga. ${ }^{31}$
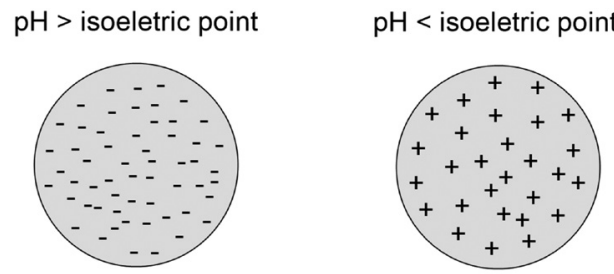

Figura 4. Comportamento das partículas de caulinita em função do $\mathrm{pH}$ do meio e do comportamento isoelétrico. As partículas adquirem carga negativa em $\mathrm{pH}$ maior que o ponto isoelétrico e positiva em $\mathrm{pH}$ menor que o ponto isoelétrico

Segundo Dollimore e Horridge, ${ }^{32}$ a caulinita exibe ponto isoelétrico próximo ao $\mathrm{pH} 5,8$. As amostras de rejeito coletadas contêm caulinita e apresentam $\mathrm{pH}$ próximo ao ponto isoelétrico (Tabela 1), sugerindo a formação dos dois tipos de cargas na extremidade das partículas. A carga resultante da interação entre as bordas acompanhada da existência da dupla camada elétrica impede a formação de aglomerados de partículas, dificultando os processos de coagulação e floculação. Para realização desse processo, é necessário que o $\mathrm{pH}$ do meio seja alterado de forma a afastar-se do ponto isoelétrico e, ao mesmo tempo, desestabilizar a dupla camada elétrica para a formação de flocos a partir de estruturas formadas face a face, face a extremidade e extremidade a extremidade.

Tabela 1. Concentração de sólidos e pH das amostras de rejeito coletadas

\begin{tabular}{ccc}
\hline Amostra & $\mathrm{pH}$ & $\begin{array}{c}\text { Concentração de } \\
\text { sólidos }(\%(w / w))\end{array}$ \\
\hline Rejeito puro & $5,76 \pm 0,04$ & 1,3 \\
\hline
\end{tabular}

\section{Teste de floculação}

A Tabela 2 exibe os valores de $\mathrm{pH}$ do meio obtidos a partir das amostras após a adição dos floculantes, e as Figuras 4, 5, 6 e 7 
Tabela 2. Valores de pH obtidos a partir das amostras no momento da adição dos floculantes

\begin{tabular}{ccccc}
\hline & \multicolumn{3}{c}{ Floculantes $-\mathrm{pH}$} \\
\hline Concentração $(\%(\mathrm{v} / \mathrm{v}))$ & $\begin{array}{c}\text { Quitosana+Acrilamida } \\
\text { (purificada) }\end{array}$ & Quitosana+Acrilamida & Quitosana & Acrilamida \\
\hline 0,3 & $6,53 \pm 0,03$ & $6,39 \pm 0,05$ & $6,35 \pm 0,08$ & $7,07 \pm 0,04$ \\
0,6 & $6,28 \pm 0,04$ & $6,08 \pm 0,03$ & $6,70 \pm 0,04$ & $7,05 \pm 0,01$ \\
1,2 & $6,37 \pm 0,02$ & $4,75 \pm 0,04$ & $4,82 \pm 0,03$ & $7,00 \pm 0,03$ \\
12,0 & $4,07 \pm 0,07$ & $3,68 \pm 0,04$ & $4,50 \pm 0,05$ & $7,07 \pm 0,02$ \\
\hline
\end{tabular}

Tabela 3. Densidade aparente dos flocos obtida das amostras após adição dos floculantes

\begin{tabular}{ccccc}
\hline \multicolumn{3}{c}{ Densidade aparente dos flocos $\left(\mathrm{g} \mathrm{cm}^{-3}\right)$} \\
\hline Concentração $(\%(\mathrm{v} / \mathrm{v}))$ & $\begin{array}{c}\text { Quitosana+Acrilamida } \\
\text { (purificada) }\end{array}$ & Quitosana+Acrilamida & Quitosana & Acrilamida \\
\hline 0,3 & $0,020 \pm 0,002$ & $* 0,068 \pm 0,005$ & $0,027 \pm 0,006$ & $0,114 \pm 0,003$ \\
0,6 & $0,029 \pm 0,003$ & $0,047 \pm 0,003$ & $0,025 \pm 0,002$ & $* 0,084 \pm 0,002$ \\
1,2 & $0,058 \pm 0,001$ & $0,043 \pm 0,004$ & $0,028 \pm 0,003$ & $* 0,213 \pm 0,005$ \\
12,0 & $0,115 \pm 0,002$ & $0,068 \pm 0,002$ & $0,028 \pm 0,004$ & $* 0,114 \pm 0,002$ \\
\hline
\end{tabular}

*suspensão apresenta alta turbidez.

apresentam as imagens retiradas das amostras a partir dos ensaios de floculação. A Tabela 3 apresenta os valores de densidade aparente dos flocos.

Observa-se a partir da Figura 1S que a turbidez das amostras e a densidade aparente dos flocos acompanham a concentração de floculante (Quitosana+Acrilamida (purificada)) adicionado. Tomando-se como referência o valor do ponto isoelétrico encontrado para as amostras de caulinita (5,7, aproximadamente) e os valores de pH encontrados para as amostras, supõe-se que nas concentrações abaixo de $12 \%(\mathrm{v} / \mathrm{v})-\mathrm{pH}$ acima do ponto isoelétrico - as partículas de caulinita adquiram carga negativa nas extremidades, podendo reagir diretamente com os cátions formados na acrilamida quando dispersa em meio aquoso, permitindo a formação de flocos através do mecanismo de ponteamento (Figura 5, mostrando a formação de pontes entre as partículas do meio e o polímero floculante utilizado). ${ }^{33,34} \mathrm{Na}$ concentração mais elevada (12\% (v/v)), a acidez elevada do meio - onde o $\mathrm{pH}$ é mais baixo que o ponto isoelétrico - favorece a formação dos dois tipos de carga na superfície das partículas de caulinita, permitindo que o processo de floculação ocorra (porém com menor eficácia), possibilitando que algumas partículas do rejeito permaneçam no sobrenadante. ${ }^{34}$ À medida que a concentração sofre elevação, os flocos aumentam em volume (diminuem a densidade), reforçando a hipótese que o processo de floculação ocorre por ponteamento e interação eletrostática. ${ }^{8,20}$

A Figura $2 \mathrm{~S}$ exibe imagens obtidas a partir de amostras de rejeito tratadas com floculante de Quitosana+Acrilamida (sem filtração). Nessas amostras a acidez do meio não foi neutralizada, permitindo a produção de floculante com excessos de íons $\mathrm{H}^{+}$. As amostras apresentaram partículas dispersas no meio e os flocos formados apresentaram menor volume que os apresentados pelas amostras purificadas. $\mathrm{O}$ excesso de cátions no meio diminui o $\mathrm{pH}$ das amostras (aumenta a acidez), alterando a dinâmica de floculação provocada pela modificação do balanço de cargas do meio. ${ }^{35}$ Nas concentrações de 12 e $1,2 \%(v / v)$, os flocos apresentam maior porosidade (verificada pela altura da coluna de sólidos), enquanto nas concentrações de 0,6 e $0,3 \%$ se observa a desestabilização da suspensão, porém, sem a formação de flocos.

As amostras de quitosana (Figura 3S) apresentaram comportamento semelhante ao apresentado pelo floculante a base de Quitosana+Acrilamida (purificada). A turbidez diminui com o aumento da concentração de polímeros. Observa-se, no entanto, que diferentemente da amostra de Quitosana+Acrilamida (purificada), a densidade aparente dos flocos não varia. As concentrações de $12 \mathrm{e}$ $1,2 \%(\mathrm{v} / \mathrm{v})$ apresentaram $\mathrm{pH}$ próximo ao ponto isoelétrico, favorecendo a formação dos dois tipos de cargas na extremidade das partículas de caulinita, dificultando assim a formação dos flocos. ${ }^{20,33,34} \mathrm{Nas}$ demais concentrações ( $\mathrm{pH}$ acima do ponto isoelétrico), as partículas apresentam carga negativa, permitindo a interação com os sítios positivos pertencentes à quitosana em meio aquoso. ${ }^{33,34}$

$\mathrm{O}$ tratamento do rejeito com acrilamida (Figura 4S) apresentou diminuição da turbidez com aumento da concentração de rejeito. As amostras apresentaram pH neutro em todas as concentrações, indicando a formação de sítios de cargas negativas nas partículas de caulinita. ${ }^{36}$ Como foi adicionado diretamente a acrilamida (monômero), a formação de flocos depende diretamente da concentração desse composto no meio. ${ }^{37} \mathrm{~A}$ acrilamida sofre

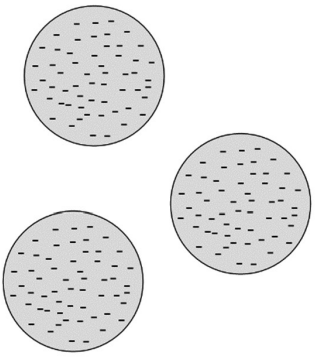

Suspended particles

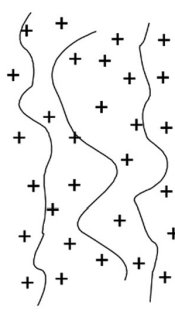

Polymer

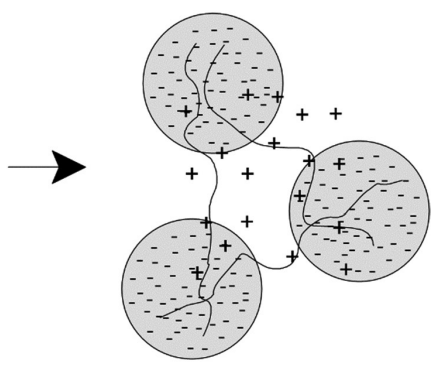

Bridging flocculation

Figura 5. Mecanismo de floculação por ponteamento em $\mathrm{pH}$ maior que o ponto isoelétrico 
protonação em meio aquoso. ${ }^{38}$ Observou-se que a formação de flocos é diretamente relacionada ao número de cátions presente no meio e, consequentemente, à concentração do floculante. ${ }^{37}$

\section{Turbidez e volume de água clarificada}

A Figura 6 apresenta o resultado dos ensaios de UV-VIS, realizado a partir da coleta do sobrenadante, e a medida da turbidez através da absorbância do meio. Os resultados reforçam os demais dados apresentados anteriormente. A concentração de $12 \%$ (v/v) exibe absorbância acima de 1,0 em todos os floculantes. Os reagentes à base de quitosana apresentaram $\mathrm{pH}$ abaixo ou próximos ao ponto isoelétrico da caulinita $(5,7)$, indicando que a turbidez pode estar relacionada à formação de cargas de sinais opostos nas extremidades das folhas de sílica e alumina. ${ }^{33,38}$ Em contrapartida, a acrilamida na concentração de $12 \%(\mathrm{v} / \mathrm{v})$ possui $\mathrm{pH}$ neutro, indicando que a alumina nesse caso adquire cargas negativas nas extremidades, tornando o processo de floculação inteiramente dependente da concentração de acrilamida. ${ }^{36}$ Como mencionado anteriormente, observa-se nesse floculante a redução da absorbância juntamente com o aumento da concentração em um meio cujo $\mathrm{pH}$ é neutro, sugerindo que a floculação (que ocorre por interação eletrostática) depende diretamente da concentração de acrilamida e o número de cátions formados em meio aquoso. ${ }^{37}$

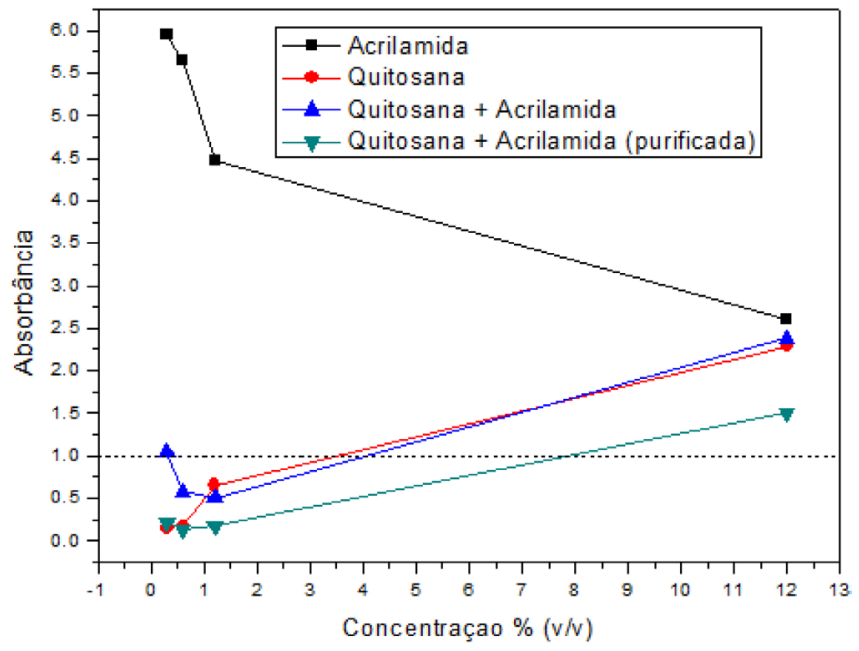

Figura 6. Ensaio de UV-VIS realizado a partir da coleta do sobrenadante das amostras utilizadas

O papel da concentração volumétrica e do tipo de floculante no processo de floculação das amostras de caulim é evidenciado a partir dos resultados, sendo importante ressaltar que a alteração da concentração não obedece a uma relação linear. Pode-se observar na Figura 6 que, para as concentrações de até 1,2\% (v/v) de floculante, a absorbância da maior parte das amostras foi menor que 1,0, indicando remoção de material suspenso e diminuição da turbidez das amostras. A enxertia de acrilamida na quitosana apresentou resultados favoráveis em relação à eficiência de floculação, além do que valores de absorbância menores foram obtidos ao utilizar o floculante na sua forma purificada.

Ademais, a floculação depende do $\mathrm{pH}$ do meio, isso porque o número e a natureza das cargas presentes na solução são responsáveis por alterar o balanço de cargas na estrutura da caulinita. Nas regiões internas ela apresenta sempre cargas negativas, porém, com intensidade variando de acordo com o pH do meio; e nas extremidades pode apresentar cargas negativas em $\mathrm{pH}$ acima do ponto isoelétrico, positivas em $\mathrm{pH}$ abaixo e ambas as cargas em $\mathrm{pH}$ igual. Em termos de eficiências de processo, as amostras de Quitosana+Acrilamida (purificada) apresentaram menor absorbância com as concentrações utilizadas.

Zhang et al. utilizaram, assim como no presente estudo, quitosana (grau de desacetilação de 96\%) enxertada com acrilamida na floculação de resíduos de caulim, alcançando absorção máxima de aproximadamente 0,06 ao aplicar uma concentração de $1,5 \mathrm{mg} \mathrm{L}^{-1}$ do floculante. ${ }^{20}$ Os autores pontuam que quanto maior o grau de desacetilação da quitosana, maior a eficiência do floculante após enxertia de acrilamida.$^{20}$ Por sua vez, Sun et al. sintetizaram o mesmo floculante (quitosana+acrilamida $\left.\left(6,0 \mathrm{mg} \mathrm{L}^{-1}\right)\right)$ pela técnica de indução de plasma, aplicando-o no tratamento de suspensão algal de baixa turbidez $(\mathrm{pH} 7,0) .{ }^{39}$ Os autores reportaram eficiências de remoção de clorofila- $\alpha$, turbidez e demanda química de oxigênio (DQO) de, respectivamente, $93,6 \%, 94,5 \%$ e 98,2\%; enquanto que a aplicação de apenas acrilamida como floculante resultou em remoções máximas para os respectivos parâmetros de $88 \%, 84 \%$ e $86 \%$, aproxidamente. ${ }^{39}$ Kang e McLaughlin testaram a atuação de quitosana e acrilamida separadamente na floculação de sedimentos de solo ricos em esmectita e caulinita. ${ }^{40}$ Nos testes com quitosana na concentração de $2,0 \mathrm{mg} \mathrm{L}^{-1}$, atingiram-se remoções de turbidez de até $40 \%$ para o sedimento rico em esmectita e $80 \%$ para aquele rico em caulinita. ${ }^{40}$ Em contrapartida, nos testes com acrilamida $\left(2,0 \mathrm{mg} \mathrm{L}^{-1}\right)$ as remoções de turbidez foram de $83 \%$ e $97 \%$ para os respectivos sedimentos. ${ }^{40}$

Ademais, a biodegradabilidade é uma importante característica relacionada à estimativa de possíveis impactos ambientais negativos oriundos da aplicação dos floculantes avaliados no presente estudo. Zhang et al. e Matica et al. destacam que a biodegradação da quitosana e seus derivados é diretamente influenciada pelo grau de desacetilação, massa molecular e modificações químicas na cadeia do polissacarídeo, uma vez que o aumento no grau de desacetilação e a redução da massa molecular elevam a sua taxa de biodegradação. ${ }^{41,42}$ Ressalta-se que a degradação biológica da quitosana resulta na formação de oligossacarídeos não tóxicos. ${ }^{42} \mathrm{~A}$ poliacrilamida e seus derivados - como o monômero acrilamida - podem ser biodegradados em até $91 \%$ (porcentagem de diminuição da massa molecular) sob condições aeróbias e anaeróbias. ${ }^{43} \mathrm{~A}$ acrilamida é uma substância tóxica para a maioria dos microrganismos, porém algumas espécies de bactérias (pertencentes aos gêneros Enterobacter sp., Rhodococcus sp. e Bacillus sp.) e fungos (Phanerochaete chrysosporium, Aspergillus oryzae, Rhodotorula sp. MBH23 e Saccharomyces cerevisiae) podem utilizá-la como fonte de carbono e nitrogênio. ${ }^{43}$ A quitosana enxertada com acrilamida, por outro lado, pode sofrer biodegradação através de mecanismos de hidrólise química e enzimática, o que reduz as ligações de - $\mathrm{OH}$ entre as moléculas de quitosana e acrilamida e, por consequência, a massa molecular do composto enxertado. ${ }^{44} \mathrm{Em}$ teste de enterro de solo (soil burial test), Basuki et al. avaliaram a biodegradação de hidrogéis sintetizados por copolimerização de quitosana e acrilamida usando radiação gama. ${ }^{44} \mathrm{O}$ estudo evidenciou uma redução considerável na massa dos hidrogéis $(\sim 75 \%)$ após quatro semanas do início do experimento, resultado da degradação do material pelos microrganismos presentes no solo. ${ }^{44}$

\section{CONCLUSÕES}

Estudou-se aqui o efeito da utilização de componentes a base de acrilamida e quitosana para o tratamento de resíduo de mineração rico em caulim. Observou-se que a enxertia de acrilamida na cadeia da quitosana pode melhorar consideravelmente o processo de floculação desse tipo resíduo. Essa melhora pode ser notada a partir das medidas de absorbância, e principalmente a partir da densidade aparente dos flocos formados. Para as concentrações volumétricas de até 1,2\% de floculante, as absorbâncias das amostras foram, em sua maior 
parte, menores que 1,0 , indicando remoção de materiais suspensos e diminuição da turbidez. Por outro lado, a concentração de $12 \%$ de floculante não apresentou resultados satisfatórios, ocasionando aumento da turbidez das amostras, com exceção do floculante à base de acrilamida

Com relação ao processo de formação das estruturas dos flocos pela acrilamida, ele se dá somente por interação eletrostática, enquanto pela quitosana enxertada, por ponteamento. Além do mais, a purificação do floculante enxertado - após neutralização da acidez e filtração - incrementou a eficiência do processo de floculação.

O estudo da interação do floculante e do meio a ser tratado é um passo importante para a solução de problemas ambientais relacionados à escassez de recursos. A retirada de partículas em suspensão com a respectiva formação de flocos depende da natureza elétrica do meio. A partir do entendimento dessa natureza das partículas do meio e do comportamento elétrico do floculante, é possível determinar condições ideais para o reaproveitamento de recursos hídricos. O trabalho evidenciou que o tratamento de rejeitos de mineração por floculação visando o reaproveitamento da água é um processo que depende das alterações elétricas que uma determinada concentração de floculante pode gerar ao meio. Por outro lado, o entendimento da estrutura química organizacional do polímero a ser utilizado como floculante se torna uma ferramenta útil para o desenvolvimento de materiais aplicados à recuperação de água em rejeitos de mineração, o que contribui para a redução dos impactos ambientais de mineradoras.

\section{MATERIAL SUPLEMENTAR}

As imagens das amostras de rejeito após adição dos floculantes utilizados neste trabalho estão disponíveis em http://quimicanova. sbq.org.br, na forma de arquivo PDF, com acesso livre.

\section{AGRADECIMENTOS}

Agradecemos à UFMG, à CAPES, à FAPEMIG e ao CNPq pelo apoio técnico e financeiro.

\section{REFERÊNCIAS}

1. Brostow, W.; Lobland, H. E. H.; Pal, S.; Singh, R. P.; J. Mater. Educ. 2009, 31, 157.

2. Kummu, M.; Guillaume, J.; de Moel, H.; Eisner, S.; Flörke, M.; Porkka, M.; Siebert, S.; Veldkamp, T. I. E.; Ward, P. J.; Sci. Rep. 2016, 6, 38495.

3. Ghernaout, D.; International Journal of Sustainable Development Research 2017, 3, 36.

4. Rajasulochana, P.; Preethy, V.; Resour.-Effic. Technol. 2016, 2, 175.

5. Ismail, I. M.; Fawzy, A. S.; Abdel-Monem, N. M.; Mahmoud, M. H.; El-Halwany, M. A.; J. Adv. Res. 2012, 3, 331.

6. Nascimento C. O. C.; Veit, M. T.; Palácio, S. M.; Gonçalves, G. C.; Fagundes-Klen, M. R.; Int. J. Chem. Eng. 2019, 2019, 8324710.

7. Zaharia, C.; IOP Conf. Ser: Mater. Sci. Eng. 2017, 227, 012138.

8. Lee, C. S.; Robinson, J.; Chong, M. F.; Process Saf. Environ. Prot. 2014, 96, 489.

9. Muruganandam, L.; Kumar, M. P. S.; Jena, A.; Gulla, S.; Godhwani, B.; IOP Conf. Ser: Mater. Sci. Eng. 2017, 263, 032006.

10. Bolto, B.; Gregory, J.; Water Res. 2007, 41, 2301.
11. Ajao, V.; Bruning, H.; Rijnaarts, H.; Temmink, H.; Chem. Eng. J. 2018, 349, 622. doi: 10.1016/j.cej.2018.05.123

12. Bajdur, W. M.; Wlodarczyk-Makula, M.; Idzikowski, A.; Desalin. Water Treat. 2016, 57, 1038.

13. Salehizadeh, H.; Yan, N.; Farnood, R.; Biotechnol. Adv. 2018, 36, 92.

14. Hasan, A.; Fatehi, P.; Sci. Rep. 2019, 9, 2672.

15. Lee, K. E.; Morad, N.; Teng, T. T.; Poh, B. T.; Chem. Eng. J. 2012, 203, 370.

16. Mahmudabadi, T. Z.; Ebrahimi, A. A.; Eslami, H.; Mokhtari, M.; Salmani, M. H.; Ghaneian, M. T.; Mohamadzadeh, M.; Pakdaman, M.; AMB Express 2018, 1, 172.

17. Yang, R.; Li, H.; Huang, M.; Yang, H.; Li, A.; Water Res. 2016, 95, 59.

18. Hargreaves, A. J.; Vale, P.; Whelan, J.; Alibard, L.; Constantino, C.; Dotro, G.; Cartmell, E.; Campo, P. Clean Technol. Environ. Policy 2018, 20, 393.

19. Buenaño, B.; Vera, E.; Aldás, M. B.; Ing. Invest. 2019, 39, 24.

20. Zhang, Y.; Jin, H.; He, P.; Adv. Polym. Technol. 2012, 31, 292.

21. Carty, W. M.; Em Ceramic Engineering and Science Proceedings; John Wiley \& Sons: New Jersey, 2001, cap. 7.

22. Plançon, A.; Giese Jr, R. F.; Snyder, R.; Drits, V. A.; Bookin, A. S.; Clays Clay Miner. 1989, 37, 203.

23. Schofield, R. K.; Samson, H. R.; Faraday Discuss. Chem. Soc. 1954, 18 , 138.

24. Hsua, S. C.; Donb, T. M.; Chiua, W. Y.; Polym. Degrad. Stab. 2002, 75, 73.

25. Brady, G. S.; Clause, H. H.; Vaccari, J. A.; Materials Handbook, $15^{\text {th }}$ ed., McGraw-Hill: New York, 2004.

26. Kim, K. M.; Kim, H. M.; Lee, W. J.; Lee, C. W.; Kim, T. I.; Lee, J. K.; Jeong, J.; Paek, S. M.; Oh, J. M.; Int. J. Nanomed. 2014, 9, 29.

27. Lowe, B. M.; Skylaris, C.; Green, N. G.; Shibuta, Y.; Sakata, T.; Jpn. J. Appl. Phys. 2018, 57, 4S.

28. Ozcelik, H. G.; Barisik, M.; Phys. Chem. Chem. Phys. 2019, 21, 7576.

29. Jepson, W. B.; Philos. Trans. R. Soc. London 1984, 311, 411.

30. Zhou, Z. H.; Clays Clay Miner. 1992, 40, 365.

31. Wang, Y. H.; Siu, W. K.; Can. Geotech. J. 2006, 43, 587.

32. Dollimore, D.; Horridge, T. A.; J. Colloid Interface Sci. 1973, 42, 581.

33. Yang, Z.; Shang, Y.; Lu, Y.; Chen, Y.; Huang, X.; Chen, A.; Jiang, Y.; Gu, W.; Qian, X.; Yang, H.; Cheng, R.; Chem. Eng. J. (Amsterdam, Neth.) 2011, 172, 287.

34. Li, S.; Gao, L.; Cao, Y.; Gui, X.; Li, Z.; Water Sci. Technol. 2016, 74, 729.

35. Zulkeflee, Z.; Aris, A. Z.; Shamsuddin, Z. H.; Yusoff, M. K.; Sci. World J. 2012, 2012, 495659.

36. Fijałkowska, G.; Wiśniewskaa, M.; Szewczuk-Karpisz, K.; Colloids Surf., A 2020, 603, 125232.

37. Daifa, M.; Shmoeli, E.; Domb, A. J.; Polym. Adv. Technol. 2019, 30, 2636.

38. Tombácz, E.; Szekeres, M.; Appl. Clay Sci. 2006, 34, 105.

39. Sun, Y.; Ren, M.; Sun, W.; Xiao, X.; Xu, Y.; Zheng, H.; Wu, H.; Liu, Z.; Zhu, H.; Environ. Technol. 2017, 40, 954.

40. Kang, J.; McLaughlin, R. A.; J. Polym. Environ. 2020, 28, 1335.

41. Zhang, H.; Neau, S. H.; Biomaterials 2001, 22, 1653.

42. Matica, A.; Menghiu, G.; Ostafe, V.; New Frontiers in Chemistry 2017, 26,75 .

43. Joshi, S. J.; Abed, R. M. M.; Environ. Processes 2017, 4, 463.

44. Basuki, K. T.; Swantomo, D.; Sigit; Sanyoto, N. T.; Adv. Mater. Res. (Durnten-Zurich, Switz.) 2015, 1112, 414. 